\title{
Supernova relic neutrino sensitivity study with 2nd Hyper-Kamiokande detector in Korea
}

\author{
Dongnyeok Yeum* ${ }^{* \dagger}$ \\ Seoul National University \\ E-mail: dnyeumesnu.ac.kr
}

\begin{abstract}
Neutrino has become a new window to our universe since the observation of neutrinos from the Sun and SN1987A. Hyper-Kamiokande (Hyper-K) is a next-generation neutrino telescope with excellent capabilities in particle physics. The detector will be a water Cherenkov detector of 260 kton water mass with $40 \%$ photo coverage. With about 10 times larger fiducial volume per detector than Super- Kamiokande(SK), sensitivities for the supernova burst and the supernova relic neutrino(SRN) searches will be greatly improved in Hyper-K. Locating the 2nd detector in Korea will enhance the sensitivity of SRN search thanks to less muon flux and spallation background in deeper $(1 \mathrm{~km})$ Korean candidate sites. In this talk, we will present our sensitivity study on SRN search for several candidate sites in Korea and the various theoretical SRN flux models.
\end{abstract}

The 39th International Conference on High Energy Physics (ICHEP2018)

4-11 July, 2018

Seoul, Korea

* Speaker.

${ }^{\dagger}$ A footnote may follow. 


\section{Introduction}

Hyper-Kamiokande(HK) is a next-generation neutrino detector. As a successor of SuperKamiokande(SK), HK consists of 2 identical cylindrical-shape water Cherenkov detector with 260 kton water mass and $40 \%$ photocoverage(40,000 PMT tubes per detector). The first detector of HK will begin its construction in April 2020 in Tochibora, Japan[1]. Korea is considered a strong candidate for the second detector, and six Korean sites are considered(Table 1). Because the heights of Korean candidate sites are over $1 \mathrm{~km}$, deeper overburden is expected than HK in Japan which is $650 \mathrm{~m}$. The height and the location of those six Korean candidate sites are shown in Table 1. Because the overburden of Korean detector(KD) is deeper than HK Japanese detector(JD), less background signal is expected for the Korean site. Also, because of the geographical separation of two detectors of HK, the more precise pointing of supernova events or other astrophysical events is expected. This is for the SRN sensitivity study to show the physics potential of HK KD.

\begin{tabular}{cccc}
\hline Site & Height $(\mathrm{m})$ & Lattitude & Longitude \\
\hline Mt. Bisul & 1084 & $35^{\circ} 43^{\circ} 00^{\circ} \mathrm{N}$ & $128^{\circ} 31^{\circ} 28^{\circ} \mathrm{E}$ \\
Mt. Bohyun & 1124 & $36^{\circ} 09^{\circ} 47^{\circ} \mathrm{N}$ & $128^{\circ} 58^{\circ} 26^{\circ} \mathrm{E}$ \\
Mt. Hwangmae & 1113 & $35^{\circ} 29^{\circ} 47^{\circ} \mathrm{N}$ & $127^{\circ} 58^{\circ} 28^{\circ} \mathrm{E}$ \\
Mt. Minjuji & 1242 & $36^{\circ} 02^{\circ} 24^{\circ} \mathrm{N}$ & $127^{\circ} 50^{\circ} 57^{\circ} \mathrm{E}$ \\
Mt. Sambong & 1186 & $35^{\circ} 26^{\circ} 52^{\circ} \mathrm{N}$ & $127^{\circ} 40^{\circ} 10^{\circ} \mathrm{E}$ \\
Mt. Unjang & 1125 & $35^{\circ} 54^{\circ} 39^{\circ} \mathrm{N}$ & $127^{\circ} 21^{\circ} 26^{\circ} \mathrm{E}$ \\
\hline
\end{tabular}

Table 1: Six KNO candidate sites.

\section{Simulation of Supernova Relic Neutrino Sensitivity}

Simulation of (i) muon flux, (ii) spallation isotope yield for HK JD and all HK KDs were done as background study. Then the ratio of spallation isotope yields of HK KD/SK or HK KD/SK was simulated. From the result of (i) and (ii), (iii) signal efficiency of spallation cut was calculated, and the SRN sensitivity was calculated from it.

Background muon flux was simulated by MUSIC[2] (Table 2). I used the surface muon flux distribution from the modified Gaisser sea level muon distribution[4], and assumed $1 \mathrm{~km}$ overburden for Korean sites. As a result, about 5 times of background muon flux is expected for HK JD while the similar amount is expected for HK KD.

Next, the ratio of spallation isotope for HK JD/SK and KD/SK was simulated(Fig. 1). This can be calculated from the spallation isotope yields calculation by FLUKA[3] combined with background muon flux result. I assumed $\mu^{+} / \mu^{-} \sim 1.3[5]$. Blue dots represent the HK JD, while others represent Korean candidate sites. Amount of spallation isotopes for HK JD is 4 times more than SK while those of Korean sites are comparable to SK.

After the ratio of spallation isotope simulation, the signal efficiency of spallation cut was calculated[6]. Table 3 shows the result of the signal efficiency of spallation cut for HK JD, Mt. Bisul, Mt. Bohyun and Mt. Sambong. I only denoted three Korean candidate sites because the 


\begin{tabular}{cccc}
\hline Site & $\begin{array}{c}\text { Muon Flux } \\
\left(10^{-7} \mathrm{~cm}^{-2} \mathrm{~s}^{-1}\right)\end{array}$ & $\begin{array}{c}\text { Average Energy } \\
(\mathrm{GeV})\end{array}$ & $\begin{array}{c}\text { Flux Ratio } \\
(\text { Site/SK })\end{array}$ \\
\hline HK (Japan) & 7.52 & 202.2 & $4.97 \pm 0.99$ \\
Mt. Bisul & 1.57 & 253.7 & $1.04 \pm 0.21$ \\
Mt. Bohyun & 1.47 & 255.9 & $0.97 \pm 0.19$ \\
Mt. Hwangmae & 1.35 & 254.8 & $0.89 \pm 0.18$ \\
Mt. Minjuji & 1.41 & 253.3 & $0.93 \pm 0.19$ \\
Mt. Sambong & 1.67 & 251.3 & $1.11 \pm 0.22$ \\
Mt. Unjang & 1.46 & 256.3 & $0.96 \pm 0.19$ \\
\hline
\end{tabular}

Table 2: Total muon flux and average energy of MC calculation for all Korean candidate sites with HK JD. The amount of background muon flux order is Mt. Hwangmae $<$ Mt. Minjuji $<$ Mt. Unjang $<$ Mt. Bohyun $<$ Mt. Bisul < Mt. Sambong. Background muon flux for Korean candidate sites (1km overburden) are as good as the current SK location and much better than HK in Japan ( $\sim 4$ times of SK).

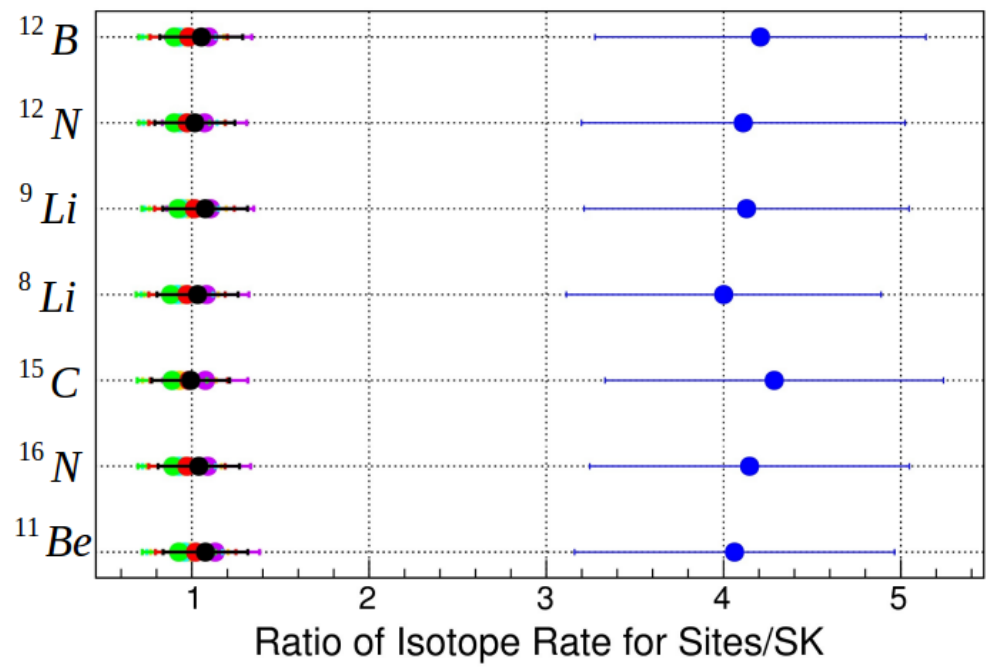

Figure 1: Ratio of spallation isotopes, HK/SK for Japanese site and Korean sites. Blue dots represents HK in Japan, while others represent Korean candidate sites. Black, red, green, cyan, violet, orange dots represent Mt. Bisul, Mt. Bohyun, Mt. Hwangmae, Mt. Minjuji, Mt. Sambong, Mt. Unjang, respectively. For HK in Japan, about 4 times of spallation isotope yield is expected than SK, while that of Korean candidate sites is comparable amount with current SK.

calculated signal efficiency for other sites (Mt. Hwangmae, Mt. Minjuji, Mt. Unjang) is the same as that of Mt. Bohyun case.

Finally, SRN sensitivity was calculated[6]. From the signal efficiency, the expected number of detected SRN events and significance for six Korean candidate sites are calculated. I also included those of JD for the case of two detectors in Korea and Japan. See Table 4 and Fig. 2. 


\begin{tabular}{lcccc}
\hline & \multicolumn{4}{c}{ Signal Efficiency of Spallation Cut (\%) } \\
\cline { 2 - 5 } Energy range & JD & Mt. Bisul & Mt. Bohyun & Mt. Sambong \\
\hline $17.5-20 \mathrm{MeV}$ & 29.1 & 70.9 & 78.9 & 68.4 \\
$20-26 \mathrm{MeV}$ & 54.7 & 84.9 & 89.6 & 81.1 \\
\hline
\end{tabular}

Table 3: Signal efficiency of spallation cut, for HK JD(Tochibora) and KNO. Other sites(Mt. Hwangmae, Mt. Minjuji, Mt. Unjang) are not shown in here because their results are same with Mt. Bohyun case.

\begin{tabular}{ccccc}
\hline Location & No. Events & $\sigma$ & Events $(+\mathrm{JD})$ & $\sigma(+\mathrm{JD})$ \\
\hline Mt. Bisul & $\sim 99$ & $\sim 5.1$ & $\sim 171$ & $\sim 6.6$ \\
Mt. Bohyun & $\sim 103$ & $\sim 5.1$ & $\sim 175$ & $\sim 6.7$ \\
Mt. Hwangmae & $\sim 103$ & $\sim 5.3$ & $\sim 175$ & $\sim 6.7$ \\
Mt. Minjuji & $\sim 103$ & $\sim 5.3$ & $\sim 175$ & $\sim 6.7$ \\
Mt. Sambong & $\sim 96$ & $\sim 5.1$ & $\sim 169$ & $\sim 6.6$ \\
Mt. Unjang & $\sim 103$ & $\sim 5.3$ & $\sim 175$ & $\sim 6.7$ \\
\hline
\end{tabular}

Table 4: Result of SRN sensitivity for KNO. The Second column is for the number of events with 10 yrs of detection ( 1 detector in $\mathrm{KNO}$ ), third column is for the significance ( 1 detector in KNO, 10 yrs), fourth column is for the number of events in $\mathrm{KD}+\mathrm{JD}(10 \mathrm{yrs})$ and the last column is for the significance of $\mathrm{KD}+\mathrm{JD}(10 \mathrm{yrs})$. For JD, $\sim 72 \mathrm{SRN}$ events and $\sim 4.2 \sigma$ is expected in $10 \mathrm{yrs}$ of detection.

\section{Summary}

Because of the deeper overburden in Korean sites, more SRN events $(\sim 72$ events in JD and $\sim 100$ events in KD, for 10 years of detection) and higher significance $(\sim 4 \sigma$ for JD and $\sim 5 \sigma$ for $\mathrm{KD}$ ) are expected for the $\mathrm{HK} \mathrm{KD}$, and also for the KD+JD. Therefore, at least in SRN case, construction of Korean detector will improve its physics potential of HK.

\section{References}

[1] K. Abe et al., Hyper-Kamiokande Design Report (2018), arXiv: 1805.04163[hep-ex].

[2] V. A. Kudryavtsev, Muon simulation codes MUSIC and MUSUN for underground physics (2008), arXiv: 0810.4635[hep-ex].

[3] A. Fasso, A. Ferrari, J. Ranft, and P.R. Sala, FLUKA: a multi-particle transport code, CERN-2005-10 (2005), INFN/TC-05/11, SLAC-R-773.

[4] T. K. Gaisser and T. Stanev, Cosmic Rays, Review of Particle Physics, edited by L. Alvarez-GaumÃl' et al., Physics Letters, B592, 228 (2004).

[5] The CMS Collaboration, Measurement of the charge ratio of atmospheric muons with the CMS detector, Phys.Lett. B 692, 83 (2010).

[6] K. Bays et al., Supernova relic neutrino search at super-Kamiokande, Phys. Rev. D 85, 052007 (2012). 